\title{
THE TYPED DOMAIN - A RECIPE FOR CREATING VIRTUAL ENTERPRISES
}

\author{
Yigal Hoffner ${ }^{1}$, Simon Field+ and Christian Facciorusso ${ }^{1}$ \\ ${ }^{1}$ IBM Research, Zurich Research Laboratory \\ e-mail: yho/cfa@zurich.ibm.com \\ +Matching Systems Ltd., SWITZERLAND \\ e-mail:spf@matchingsystems.com
}

\begin{abstract}
This paper presents an approach for describing inter-organisational relationships, based on the concept of the typed domain, which helps establish and enact successful relationships between partner organisations in specific domains. The typed domain consists of the relationship life cycle, projections and their documents, and domain building blocks of different granularity from which the relationship can be described, established and built. The relationships among the projections and the mappings among their various documents and related agreements can be exploited to structure and simplify the negotiation between partners. Furthermore, the mappings can be used to translate an agreement reached in one projection to agreements in other projections. This can be achieved using the typed domain as the context for the transformations and where necessary, involving further negotiation cycles.
\end{abstract}

\section{INTRODUCTION}

In most business domains, common understanding and conventions evolve over time as to the manner in which business is expected to be transacted within the domain. In so called 'traditional' business domains, the transactions are based on a certain degree of trust brought about by familiarity among the players and the assumption that they all possess common domain knowledge. This cannot be assumed in the case of electronic markets and virtual enterprises. In the first place, the players are no longer restricted by the same physical presence as in traditional markets, and general consensus concerning service definitions, what constitutes an agreement and what are the penalties for non-delivery or bad quality of service are not necessarily clear. Moreover, if business processes are provided as web services, access to them has to be carefully controlled to prevent unwanted interactions from unauthorised parties. A substitute for the traditional domain concepts therefore is needed for its virtual equivalent to have any chances of commercial success.

We refer to the electronic equivalent of the traditional business domain as the 'typed' and 'closed' e-domain. The 'closeness' and 'typedness' concepts are based 
on the principle of no surprise and act as substitutes for the notion of familiarity of the traditional business-domain. The e-domain is regarded as the basis for virtual markets and virtual enterprises.

The typed domain provides a common language that is used to define one or more possible relationships - the associated expectations from the relationship, the interactions between the parties, as well as the obligations and responsibilities associated with the exchanges between the two organisations. It fosters a common shared understanding of what the domain vocabulary means among the domain members, and ensures that no interactions with unexpected operations, data exchanges or exceptions can take place between the parties. Applying the distributed systems principle of 'no surprise' to the e-domain requires a full definition of many things: the initial offers and counter-offers in the negotiation process and matchmaking processes, the agreements and contracts, services and clients, monitoring and supervision. In short, it encompasses all the aspects that concern the establishment, enactment, termination and post-processing of a business relationship. The 'closed' domain restricts access to information, to facilities and to members of the domain and therefore complements the concept of the 'typed' domain.

Although we regard the typed and the closed domain concepts as a pre-requisite to creating the electronic world that binds the parties of a virtual enterprise together, the concept of the closed domain lies outside the scope of this paper and the remainder of the paper concentrates on the concept of the typed domain. The paper describes the concept and exposes its constituent parts, showing how the distributed systems type-safe binding principle can be generalised to encompass all the aspects that concern the establishment, enactment and dissolution of a business relationship.

The complexity of inter-organisational relationships leads us to view the edomain from four different views or projections, each centred on a different set of concerns and associated roles. The relationships among the projections and their various documents and agreements can be exploited to structure and simplify the negotiations between service provider and consumer organisations. Furthermore, the typed domain and its projections can act as a context in which agreements reached in one projection can be translated to agreements in other projections. Where necessary, such transformations may involve further negotiation cycles between the two parties. Thus, the projections and the transformations among them can form an important basis for the structuring of the negotiation process and the decision making that surrounds the process of service consumer-provider relationship establishment. Different schemes combining negotiations in one or more projection and then translating to the corresponding agreement in another projection are possible. The richness of the possible relationships in a typed domain and the granularity of the domain building blocks influence the complexity of those translations.

\section{THE TYPED DOMAIN}

The 'typed' domain is based on the "no surprise" principle - ensuring that no unexpected relationships are established, no unwanted interactions, operation invocations, data exchanges or exceptions can take place between the parties. The 
principle of 'no surprise' is a generalisation of the distributed systems' type-safe binding idea (Open Distributed processing standards (ODP, 1995), CORBA standards (OMG, 1996) and web services (WS, 2002)). It ensures that possible interactions between the parties are fully defined prior to run-time and also that any dynamic bindings that may take place during operation are type safe. The generalisation and application of this principle to the domain requires a full definition of the possible relationships that can be established. To accomplish this requires an inordinate amount of information for describing inter-organisational relationships to be agreed a-priori. The intricate nature and quantity of this information requires a special approach for dealing with this complexity. The typed domain provides such an approach through the following:

1. Life Cycle stages: provide a description of the steps that organisations have to engage in to form, enact, terminate and post-process a relationship.

2. Projections and their documents: provide a way of separating the concerns surrounding a relationship into four areas.

3. Domain building blocks: provide components of different granularity from which the relationship can be described and built, ranging from monolithic templates to finer granularity components with composition rules.

The following sections provide an overview of the typed domain constituent parts.

\subsection{Overview of the relationship life cycle}

A relationship life cycle is a description of the steps that two organisations have to go through in order to establish, enact, maintain and manage the desired business relationship between them (Milosevic, 1995) (Dignum, 2001) (Vetter, 2001). We regard an electronic version of the life cycle as consisting of the following phases (Hoffner, 2003):

- Establish relationship: this entails finding a potential partner through advertising, querying, matchmaking, exchanging information, negotiating and agreeing. This should result in the establishment of a client-service agreement/contract.

- Deploy service and client: the agreement is used as a blue-print for creating or configuring the service, client and related infrastructures. Once the components are in place, they can be linked.

- Operate service and client: this entails the provision and consumption of core and if required, of auxiliary services.

- Terminate the relationship: this entails agreement on relationship termination and the dismantling of the relationship.

- Post-process the relationship: this entails preparing an audit report, evaluating the relationship and providing feedback.

Instances of the typed domain in specific application and commercial areas will exhibit different flavours of the life cycle, possibly using different sub-sets and orderings of the stages and sub-stages.

\subsection{Overview of the projections and their documents}

The complexity of the life cycle stages is too big to be described in a monolithic form. We therefore break up the description of the life cycle into different views or projections as shown in Figure 1.1. The projections are interrelated but not in a layered or containment fashion, rather as four different points of view of the same 
relationship. The projections help structure and separate the concerns surrounding the typed domain into manageable parts. Each projection is also associated with a different role or roles as will be shown later, with the main responsibility for the projection lies with the role holder. The typed domain describes the possible relationships between organisations in terms of the different types of entities that exist in each projection and the relations between them.

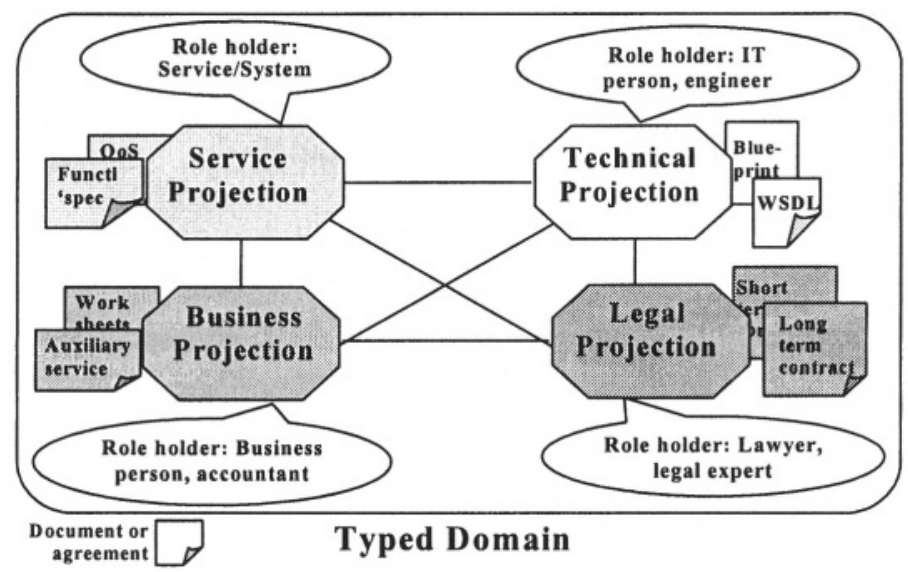

Figure 1.1: The four projections that describe the entire relationship between two organisations, each from a different perspective, help structure the information of the typed domain.

Each projection has a representation of the relationship from its perspective covering all the information necessary for carrying out the life cycle:

Technical projection: This projection specifies how and where the service will be provided and consumed. It describes the possible interactions in terms of invocations and replies, the data carried, the protocols used and the destinations. It contains a detailed engineering specification of the points of contact between the client and service sides (and of any auxiliary services that are needed in addition to the core service).

The role holder associated with this projection is the IT person whose responsibility is to create and maintain the information systems necessary to support the relationship and ensure that its availability, performance, security, etc., are kept to standard. The role holder will also be responsible for ensuring that the computing resources are utilised properly and efficiently.

Examples of projection documents are: Interface or port and binding information (protocols and addresses) using IDL (OMG, 1996) or WSDL (WSDL, 2001), describing service interfaces, as well as management, monitoring and termination interfaces. The interface/port and binding information format will depend on the specific technology used: WS SOAP, CORBA IDL and IOR, Message Passing (JMS, MQseries), EDI, RMI, email, telephone or ordinary mail.

Service projection: This projection specifies what can be delivered and consumed in functional terms. It contains a functional and behavioural description of services in terms of invariant attributes, global constraints, operation pre-conditions, effects or post-conditions. It specifies the permissible sequencing or choreography of 
interactions expressed for example in BPEL (BPEL, 2002)). It contains Quality of Service (QoS) attributes, statements and requirements, describing what the service can do and how it can be consumed. Likewise, the expectations from the consumer are also specified in terms of behaviour and quality.

The role holder associated with this projection is the systems and service person whose responsibility is to ensure the proper execution of services and their QoS. Customer assistance and addressing customer complaints is also under their responsibility.

Examples of projection documents: service (functionality/behaviour) descriptions with QoS indications, permissible sequencing specification.

Business projection: This projection specifies what will be delivered in terms of consideration - tying together service consumption and remuneration aspects of the relationship. It looks at the relationship from a business point of view - what does each side provide and consume. This usually involves the notion of consideration usually defined in monetary terms. Since the concern is financial, there will be a description of how the provision and consumption can be observed, measured and verified. It is at this level that the relationship between the work performed or product delivered will be related to costing. The projection is therefore likely to involve auxiliary services for measuring, costing, billing, paying and receipt handling. The projection may also include statements about what penalties are incurred if promises are not kept.

At this level of description, there is also a specification of the termination of the relationship: what defines a satisfactory enactment of the contract; how is the termination agreement conveyed to the other party, and ultimately - what is the procedure for the termination (Hoffner, 2003).

The role holder associated with this projection is the businessperson or accountant whose responsibility includes the commercial aspects of the relationship - profitability, return for investment and customer satisfaction from the point of view of quality and cost effectiveness.

Examples of projection documents: full business description, executive summary, business plan, profit expectations, work sheets, audits, failure reports.

Legal projection: This projection outlines the promises made by the two parties in a legal framework, specifying what recourse to the law the parties have in case of disputes. It deals with legal issues such as scoping of the relationship, exclusions, legal notices in case of problems, penalties, arbitration. The projection describes what are the promises, obligations and liabilities of the parties and specifies what happens if there are any deviations from the promises or disputes.

The role holder associated with this projection is the lawyer or legal expert whose responsibility includes ensuring that the scope and liabilities are limited, that when problems occur the organisation is covered from unreasonable demands, that no infringements of IP or copy rights takes place, etc..

Examples of projection documents are umbrella contracts - short term contracts within the context of a long term one, normal contracts, documents of understanding (outlining the principles of an agreement).

\subsection{Overview of the domain Building Blocks}

The components that the domain has, whether actual objects or descriptions of 
objects, protocols, QoS can consist of units of different granularity:

Monolithic document and agreement templates: complete templates that require no assembly from finer-grained clauses, but are populated with the relevant information from the business agreement. This has the advantage of simplicity, but cannot cope with structural variation among documents and agreements that need to share the same template.

Compositional documents: instead of complete templates, the domain consists of clause templates that have to be assembled into complete documents and agreements and populated with the relevant information from the business agreement. In such a case, there is a need to define:

- Building blocks: template components or clauses of finer granularity than the monolithic templates.

- Composition rules: component or clause selection and exclusion rules that will use the information from the business agreement to determine which clauses should be included or excluded.

- Ordering, consistency checks and restrictions: post clause selection processing to see if the assembled document or agreement is valid in the domain.

Hybrid documents and agreements: using a combination of a small number of monolithic templates together with a number of templates of smaller granularity that can be assembled in different ways.

\section{RELATIONS AMONG PROJECTIONS AND THEIR DOCUMENTS}

The typed domain definition consists of a full description of all possible and allowed relationships in such a manner that ensures that only safe relationships can be built within its boundaries. A typed domain provides one or both of the following:

- Exhaustive (full) specification of possible relationships: The full definition(s) of all that can take place between the two parties. We refer to such definitions as monolithic - each description specifies a relationship type.

- Generative specification of possible relationships: A way to generate relationship possibilities (from building blocks of finer granularity than the monolithic types, composition rules and restrictions) that describe what can take place between the two parties.

Each possible relationship in a typed domain is fully prescribed by the four projections. Each projection is likely to contain different kinds of agreements and documents that describe different aspects of the same permitted relationship or relationships.

For example, one or more service types, offering different flavours of the same business, may support a specific business objective. It may also be possible that for the same type of business, different remuneration schemes exist and different auxiliary service types support these. For example, for the same business type, the remuneration service may be offered in different computerised forms or alternatively through conventional banking procedures outside the computer system.

The same service may be provided through different types of interfaces, possibly using different binding schemes, i.e. protocols and addresses. For example, the same service may be offered as a CORBA or WS implementation or alternatively 
through a message passing infrastructure. This demonstrates a connection between a service projection description and the related descriptions in the technical projection.

Other aspects of the relationship described in the legal projection, may also provide some degrees of freedom - the same type of business may be enveloped with different contracts depending on the legal context referred to and the specific promises made. For example, whether a service is offered nationally or internationally, and depending on legal restrictions and scoping given with it and the standing of the provider organisation (sole trader, second source, partnership, private or public Ltd., etc.).

The possibilities and limitations that decisions in one projection will impose on others must be specified in a set of relationships. The typed domain acts as a constraint, not just on the total set of information, but also on the mapping between the different documents and agreements in the domain. This inter-dependence among the projections brings with it considerable complexity but also carries some distinct advantages.

Many of the documents and agreements across domains will share common information, often across different projections, and at different stages of the Life cycle. Completion of a document or agreement may therefore spawn partial completion of documents or agreements elsewhere in the domain, reducing the amount of information required at later stages, or in other projections.

The granularity of the domain building blocks will affect the complexity of the descriptions in the projections and the complexity of the relationships between the projections. It may be that the mappings between the descriptions in the projections can be specified at the monolithic type level, so that the connection between different business, service, technical and legal types can be described with a relationship type. For the same type of relationship in a specific domain, there may be different options or degrees of freedom associated in the other projections.

A finer granularity of building blocks, for example, where many possible contract clauses are offered by a service provider, will entail a different mapping from the business to the legal projections. Some of these may not be appropriate for all service offerings, and once an agreement has been reached in the service projection, specifying an individual service with agreed configuration options, the choice of possible clauses that can contribute to the service contract in the legal projection may be severely limited. Indeed, there may be no remaining choices regarding the contract, the choice of service effectively dictating the contract that must be agreed.

The following section demonstrates how the typed domain and its projections can help address the complexity of establishing a relationship between organisations.

\section{USING THE PROJECTIONS FOR NEGOTIATIONS}

The relationships among the projections and the mappings among their documents and agreements can be exploited in:

- Negotiation: negotiation entails a structured navigation through a space of possible relationships and configurations, pruning the options that are 
unacceptable to one or both parties. The mappings can thus help structure and simplify the negotiation between a service provider and consumer organisations.

- Translation: transforming an agreement reached in one projection to agreements in other projections by relying on the pre-specified mappings among them. This can be achieved using the typed domain as the context for the transformations and, where necessary, involving further negotiation cycles.

\subsection{Structuring the negotiation process}

In a typed domain, the mapping among the projections is fully specified - every relationship specified in the domain will have its description in each of the projections and these descriptions will be related to each other. Selecting a type of relationship in one projection and fixing some of its details, is likely to reduce the options in the related projections that will fit the entire relationship.

On occasions, it is easier to isolate those views from each other and treat them (as much as is possible) separately. This often simplifies the negotiation process; thus, the business details may be discussed in isolation of the exact technical details, or the service may be discussed without referring to the business aspects. Similarly, business agreements are often done without explicit or direct reference to legal issues. The assumption is that those can be sorted out at another point. The assumption behind the separation is that there is a common model (of the domain of discourse) that allows assumptions to be made and provides the assurance that in spite of the separation, the entire relationship will work.

It appears from our experience that the starting point for the process of establishing a relationship is in the business projection. The rationale for this is that the driving force behind relationships is first and foremost a commercial one and not a technical, service or legal one. Thus, the projections and the transformations between them are well positioned to be exploited in both the structuring of the negotiation process and the decision making that surrounds the process of service consumer-provider relationship establishment.

The four projections facilitate some flexibility regarding the progression through the life cycle stages. Whilst it may be the case that all four projections progress through the stages in parallel, it is also possible, and perhaps more likely, that each projection runs through the life cycle at a different pace. For example, establishing agreement in the Business Projection (that the two organisations will benefit from a business relationship, and wish to reach an agreement), may precede the other projections.

Progress through the life cycle in the different projections at different speeds can have an advantage over a more synchronised progression. Decisions made in the stages completed early on may have the effect of restricting the range of options to be considered in later stages, or in other projections. For example, reaching an agreement in the Business Projection will probably limit the options that need to be considered in the Service Projection and once a specific service has been agreed, the options to be considered and selected in the Technical Projection may in turn be reduced.

In the establishment stage of the life cycle, there are two general approaches for fully specifying a relationship in all projections:

1. Carry out the negotiation process (with matchmaking responsible for different degrees of it) with respect to all the projections at the same time. 
2. Carry out the negotiation process with respect to a single or a mixture or some of the projections, then use the agreement reached to prune the options in the other projections and generate the agreement in the other projections.

This second approach has the advantage that having determined the type and details of the relationship in one projection, it is clear that there is at least one counter-part in each of the other projections that may satisfy the requirements of the other party and vice versa. Fixing the type and details of an agreement or document in one projection, greatly reduces the number of options and therefore exchange of information and decisions that both sides have to make concerning the agreements and documents in other projections. A likely scenario is to negotiate the business agreement and then transform it into the other agreements. With each transformation, the degrees of freedom will be reduced, leaving less options and decisions to be made.

The agreement stage in the life cycle does have a particular importance, culminating in the establishment of a contract. It is highly unlikely that a contract can be established until at least some level of agreement has been reached in all four projections, though that may, for example, be "an agreement to reach an agreement at a later date" in the Technical Projection. It is also unlikely that the stages beyond the establishment of the contract will proceed in any of the projections until the contract has been established. Establishment of the contract thus acts as a point of synchronisation, or checkpoint, across all projections.

\subsection{Transforming documents from projection to projection}

If the granularity of the projection building blocks is coarse, a correct type selection based on the mapping among the projections will be sufficient to make the transformation from the description of the relationship in one projection to another. Where the granularity is finer, the complexity of the description in one projection will have to be mirrored in the others and the mapping will require complex rules.

A detailed example of a business to legal agreement (contract) transformation [Field 04], based on a fine granularity of service description and contract clauses, demonstrates how a document in one projection can be transformed to a document in another projection. In this specific example of derivative trading, a business agreement expressed using the Financial Products Mark-up Language (FpML ${ }^{\mathrm{TM}}$, 2003), is transformed into the legal confirmation contract in the context of its typed derivative trading domain.

The process looks at the description of the business agreement, analyses it and then determines what the contract should look like. When transforming an agreement to a contract, there may be a need for additional information from one or both of the parties. The process itself will differ largely depending on what are the constituent parts or building blocks of the contract assembly defined in the domain.

This method can be generalised so that other agreement transformations both within and between the projections are possible.

\section{CONCLUSIONS AND FUTURE WORK}

We base our concept of the typed domain and its projections on the belief that a complex relationship between two organisations requires more than a single point of 
view, encompassing technical, service, business and legal projections. Within the relationship life cycle, it is necessary to reach agreement at all of the projections before configuration and instantiation of the components can take place and enactment of the agreements be carried out.

There are many open questions concerning the typed domain we propose in this paper. For example - how can any rules be attached to the links among the projections, so that when one type of document in a specific projection is selected, the appropriate (and still relevant) types in the other projections are also selected, while the inappropriate ones are discarded.

We have stated that in our experience a likely starting point for the process of establishing a relationship is in the business projection. However, it is not entirely clear how the life cycle should be managed - whether other factors from the other projections should be brought in at the beginning or at a later stage.

With respect to the granularity of the domain building blocks, initially, domains with monolithic templates and types should be looked at. An important open question concerns finding what building blocks of smaller granularity than the monolithic templates and types should be used. Building blocks, rules and mechanisms for composition must then be specified, and ways of checking the validity of these compositions must be developed.

\section{REFERENCES}

1. Hoffner Y. and Field S., Dynamic Contract Generation for Dynamic Business Relationships, in "Virtual Enterprise Integration: Technological and Organizational Perspectives", Editors G. Putnik and M. Cunha, IDEA Group Inc. 2004.

2. BPEL, Business Process Execution Language for Web Services, Version 1.0, http://www106.ibm.com/developerworks/library/ws-bpel/, 31 July 2002.

3. Dignum F. "Agents, Markets, Institutions and Protocols." In Agent Mediated Electronic Commerce, The European AgentLink Perspective, Dignum F. \& Sierra C. Editors, Lecture Notes in Artificial Intelligence Vol. 1991. Berlin Heidelberg: Springer Verlag, 2001.

4. Field S. and Hoffner Y. "In Search of the Right Partner", in Collaborative Business Ecosystems and Virtual Enterprises, PRO-VE'02, 3rd IFIP Working Conference on Infrastructures for Virtual Enterprises, Editor: Luis Camarinha-Matos, Kluwer Academic Publishers, May 2002.

5. Field S. and Hoffner Y. "Dynamic Contract Generation for Dynamic Business Relationships", Chapter in "Virtual Enterprise Integration: Technological and Organizational Perspectives", Editors: G. D. Putnik, and M. M. Cunha,, IDEA Group Publishers, to appear in July 2004.

6. FpML ${ }^{\mathrm{TM}}$ (2003). The XML standard for saps, derivatives and structured products. Retrieved from http://www.fpml.org/

7. Hoffner Y, Field S, Grefen P, Ludwig H. "Contract-driven creation and operation of virtual enterprises". Computer Networks, The International Journal of Computer and Telecommunications Networking,North Holland, Volume 37, pp. 111-136, September 2001.

8. Hoffner, Y. The e-Business on Demand Life Cycle, PRO-VE'03, IFIP Working Conference on VIRTUAL ENTERPRISES, Lugano 29-30 September 2003.

9. Milosevic Z, Berry A, Bond A, Raymond, K. "Supporting Business Contracts in Open Distributed Systems". In Proceedings of the Second International Workshop on Services in Open Distributed Processing (SDNE95), Whistler, Canada, June 1995.

10. ODP, "Open Distributed Processing Reference Model". ISO/IEC 10476, ITU-T Recommendation X.900, Parts $1-3,1995$.

11. OMG, Object Management Group and X/Open Standard: "CORBA Trading Object Service", Document orbos/96-05-6, 1996.

12. OMG, Object Management Group, "CORBA 3.0: Common Object Request Broker Specification". 2003. http://www.omg.org/technology/documents/formal/corba_2.htm. 